\title{
Exploring Millimeter-Wave Network Scenarios with Ray-tracing based Simulations in mmTrace
}

\author{
Daniel Steinmetzer \\ TU Darmstadt, Germany \\ dsteinmetzer@seemoo.tu-darmstadt.de
}

\author{
Jiska Classen \\ TU Darmstadt, Germany \\ jclassen@seemoo.tu-darmstadt.de
}

\author{
Matthias Hollick \\ TU Darmstadt, Germany \\ mhollick@seemoo.tu-darmstadt.de
}

\begin{abstract}
The advantages of mm-wave communication technology with highly directional transmissions enable high data rates in dense wireless networks. Even though first consumer hardware is already available, specific propagation effects are not yet well understood. Analyses of wireless network behavior with mm-wave communication links are required to design efficient protocols. In this work, we consider three well-known problem scenarios in wireless networks in our ray-tracing based channel simulation framework mmTrace. Our results indicate that $\mathbf{m m}$ waves exhibit advantages to block interferences but also raise the question of optimal antenna alignment. Simulations as ours highlight the importance of $\mathrm{mm}$-wave propagation models as network behavior differs from common considerations.
\end{abstract}

\section{INTRODUCTION}

Millimeter-wave (mm-wave) communication systems address the demands of $5 \mathrm{G}$ networks and will be available in many devices soon ${ }^{1}$. With directional antennas, signals are steered directly towards their destination [1]. However, propagation effects are different to those in common communication technologies; reflection and blockage have a strong influence but diffraction and scattering are negligible. To understand the specialties of mm-waves, precise propagation models that encounter these effects are needed.

With mmTrace [2], we propose a simulation framework predicting channel characteristics in specific environments with multiple transceivers. Considering different antenna radiation characteristics and applying the image-based raytracing approach enables an analysis of interference and locationdependent signal characteristics in various $2 \mathrm{D}$ environments with an arbitrary number of reflecting and blocking obstacles. As shown in [2], mmTrace achieves a precision to predict the signal strength and delay spread comparable to that of common statistical models [3]. The advantage of mmTrace is its capability to adapt to different environments and deal with multiple transceivers in parallel. The MATLAB implementation of mmTrace is publicly available [4] and supports to investigate various aspects of $\mathrm{mm}$-wave propagation.

In this work, we consider three well-known problems of wireless networks and analyze their impact on mm-wave networks with mmTrace. In particular, we encounter (1) the hidden node problem with two transmitters that cause interference at a single receiver, (2) the problem of concurrent transmissions, where multiple transceiver pairs distort

\footnotetext{
${ }^{1}$ At Consumer Electronics Show (CES) 2016, device vendors announced IEEE 802.11ad compatible access points and mobile phones to appear in 2016.
}

each other, and (3) the threat of nomadic eavesdroppers that aim to overhear transmissions from arbitrary positions. Our simulations show that the hidden node problem becomes superficial for narrow beams and perpendicular node arrangement. Concurrent transmissions lead to diverse interference levels and only appear manageable with low beamwidth. The success of nomadic eavesdropping depends on the environment and antenna settings but generally becomes more challenging than in common wireless networks. These results outline the advantages of mm-waves, but also emphasize the challenge of finding optimal antenna alignments, which is vital to design reliable and efficient mm-wave communication protocols.

In the remainder of this paper, we analyze mm-wave network scenarios in simulation with mmTrace in Section II, and summarize and conclude our work in Section III.

\section{NeTWORK ScEnARIOS}

In this section, we conduct simulations of the hidden node problem, concurrent transmissions, and nomadic eavesdropping with mmTrace. We assume a room of size $4.5 \times 3.0 \mathrm{~m}$, a transmit power of $10 \mathrm{~mW}$, an arbitrary noise power of $-100 \mathrm{~dB}$, and a distance between transceivers of $2 \mathrm{~m}$ throughout all scenarios. In the following, we describe the scenarios in detail and discuss their results and impacts.

\section{A. Hidden Node Problem}

Hidden nodes are a well known problem in wireless networks. Consider three nodes A, B, and C, where B communicates with $\mathrm{A}$ and $\mathrm{C}$, but $\mathrm{A}$ and $\mathrm{C}$ are not aware of each other as illustrated in Figure 1a. In omni-directional networks this complicates carrier sensing and B might receive nothing but interference. The directionality in mm-wave networks makes even more unlikely that nodes sense each other. However, the impact highly depends on offset angles between the transmitting nodes, as shown by our simulation results in Figure 2a, where B is oriented towards A. The SINR is low for small and very high offset angles between A and C. The drop at high offset around $180^{\circ}$ originates in environmental reflections. For perpendicular alignment of A and C, the SINR achieves its maximum, and only few interferences affect the communication. Furthermore, we observe that low beamwidths lead to high SINRs. Highly directional transmission links not only provide the highest signal gain, but also effectively withstand unwanted interferences. 


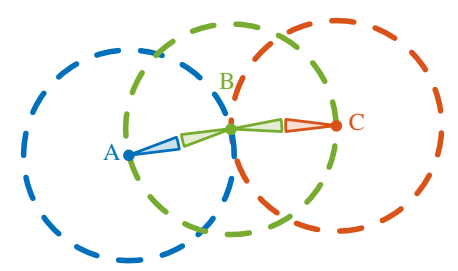

(a) Hidden Node Problem

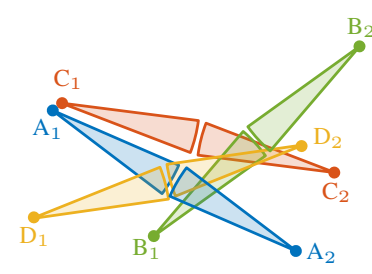

(b) Concurrent Transmissions

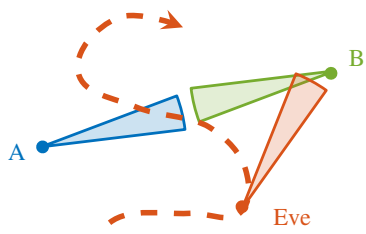

(c) Nomadic eavesdropping

Fig. 1. Illustration of network scenarios with multiple transmitters and receivers.

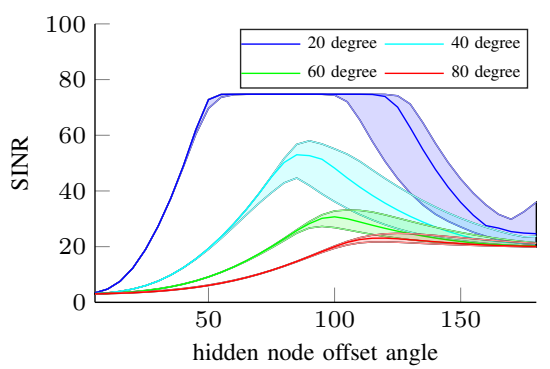

(a) Hidden Node Problem

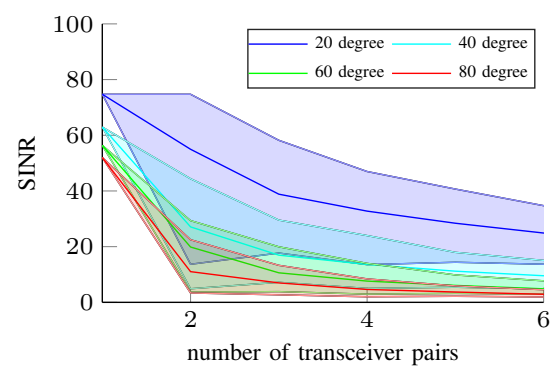

(b) Concurrent Transmissions

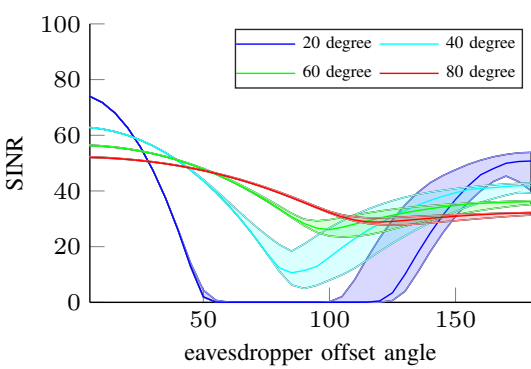

(c) Nomadic eavesdropping

Fig. 2. Simulations results of network scenarios showing the SINR median and $90 \%$ confidence bounds after 200 repetitions.

\section{B. Concurrent Transmissions}

Multiple concurrent transmissions in range of each other cause interferences and decrease throughput. This is one of the most limiting factors in omni-directional networks. Based on directionality, mm-wave systems allow for multiple transmissions at the same time. We distribute pairs of transceivers as illustrated in Figure 1b and analyze the average interference with varying number of concurrent transmissions. Our results indicate that highly directional antennas allow for a couple of transmissions. However, the beamwidth remains the limiting factor: wide antenna beams are prone to interferences. This effect depends on the relative alignment of the transmissions and results in high variances as shown in Figure 2b. Overall, the possibility of concurrent transmissions in mm-wave systems is advantageous, even though narrow beams are required.

\section{Nomadic Eavesdropping}

Omni-directional transmissions are easy to eavesdrop; attackers only have to reside in range. In mm-wave systems, eavesdroppers are assumed to be unable to overhear a transmission from outside the signal beam, which already turned out to be naive as experimentally shown in [5]. Environmental reflections significantly increase the signal strength at distinct locations in the environment and facilitate eavesdropping. We analyze the SINR at circular aligned receivers around a transmitter. This represents a nomadic eavesdropper (Figure 1c) with fixed distance. Our results, as shown in Figure 2c, outline that good eavesdropping chances exist close to the direct transmission orientation or at the opposite direction where strong environmental reflections occur. Moreover, narrow beams increase the SINR at low offsets but complicate eavesdropping from perpendicular directions. The angular dependencies relax with increasing beamwidth. In general, eavesdropping on wide beam transmissions is feasible from arbitrary positions as reflections spread the signal throughout the environment.

\section{CONCLUSION}

Predicting mm-wave network behavior is challenging as propagation effects are different to those of common wireless networks. However, as scattering and diffractions are negligible, raytracing becomes a suitable tool to predict signal propagation. In this work, we use our ray-tracing based channel simulator mmTrace to analyze the impact of three well-known problem scenarios on mm-wave networks. We show that the hidden node problem becomes an important aspect of mmwave networks, concurrent transmissions are advantageous but limited by beamwidth, and eavesdropping highly depends on the antenna beamwidth. These scenarios cover only a subset of simulation possibilities in mmTrace, but sufficiently emphasize the special behavior of mm-wave propagation that should be considered in mm-wave network design.

\section{ACKNOWLEDGMENT}

This work has been funded by the DFG within CROSSING, the BMBF and the State of Hesse within CRISP-DA, and the Hessian LOEWE excellence initiative within CASED.

\section{REFERENCES}

[1] T. Nitsche, C. Cordeiro, A. B. Flores, E. W. Knightly, E. Perahia, and J. C. Widmer, "IEEE 802.11ad: directional $60 \mathrm{GHz}$ communication for multiGigabit-per-second Wi-Fi,” IEEE Communications Magazine, vol. 52, no. 12, pp. 132-141, Dec. 2014.

[2] D. Steinmetzer, J. Classen, and M. Hollick, "mmTrace: modeling millimeter-wave indoor propagation with image-based ray-tracing," in Millimeter-wave Networking Workshop (mmNet'16), San Francisco, USA, Apr. 2016.

[3] A. Maltsev, "Channel models for $60 \mathrm{GHz}$ WLAN systems," Tech. Rep. doc.: IEEE 802.11-09/0334r8, 2010.

[4] D. Steinmetzer. (2016) mmTrace: ray-tracing based millimeterwave propagation simulation. [Online]. Available: https://github.com/ seemoo-lab/mmTrace

[5] D. Steinmetzer, J. Chen, J. Classen, E. Knightly, and M. Hollick, "Eavesdropping with periscopes: experimental security analysis of highly directional millimeter waves," in Communications and Network Security (CNS), IEEE Conference on, Sept 2015, pp. 335-343. 